\title{
SOME QUANTITATIVE ASPECTS OF THE BINDING OF QUINIDINE AND RELATED QUINOLINE COMPOUNDS BY HUMAN SERUM ALBUMIN *
}

\author{
By HADLEY L. CONN, JR. AND ROBERT J. LUCHI $\dagger$ \\ (From the Robinette Foundation, Department of Medicine, University of Pennsylvania School \\ of Medicine, Philadelphia, Pa.)
}

(Submitted for publication August 18, 1960; accepted November 21, 1960)

The interaction of quinidine with various body proteins may well be the fundamental process through which quinidine exerts its many effects. For example, quinidine binding with membrane lipoprotein may lead to decreased membrane permeability to passive ion movement. This permeability change has been held responsible for the quinidine-induced alterations in sodium and potassium exchange in cardiac muscle $(1-4)$. Quinidine binding with critical enzyme proteins may be responsible for the alterations in intermediary metabolism which have been ascribed to quinidine (5). In our experience, glucose-6-phosphate dehydrogenase activity is reduced in vitro by quinidine. Finally, the known binding of quinidine to albumin may protect the cell against the action of quinidine by making less drug available at cellular sites. The nature of the interaction with protein of quinidine and of other compounds having a similar chemical structure seemed, therefore, to warrant an investigation.

Albumin is readily available in adequate amounts and in relative purity. As noted above, it is known to complex with quinidine. Further, the state of the quinidine-albumin interaction might be considered as a prototype of the "protective" form and perhaps characteristic of all forms of quinidine binding to protein. For these reasons our initial investigations were concerned with the reactions of quinidine and related quinoline compounds with albumin. Experiments were designed to permit determination of the stoichiometry, the association constant, and the energies of binding for the reactions; and to show the contri-

* This study was supported by grants from the Atomic Energy Commission and the American Heart Association.

$\dagger$ Supported in part by United States Public Health Service Grant H5663 and a grant from the Pharmaceutical Manufacturers Association. butions to binding by the various reactive sites or areas in the quinidine molecule.

\section{METHODS}

A dialysis equilibrium technique was used to evaluate the in vitro binding of quinidine and of similar chemicals to human serum albumin. ${ }^{1}$ A limited investigation of quinidine binding to human $\gamma$-globulin was carried out in the same manner. ${ }^{2}$ A cellulose dialysis membrane ${ }^{3}$ was prepared by an initial wash with 1 per cent sodium carbonate and a subsequent wash with distilled water. Twenty $\mathrm{ml}$ of $0.2 \mathrm{M}$ phosphate buffer solution containing $250 \mathrm{mg}$ of human serum albumin was placed in a dialysis bag. Fifty $\mathrm{ml}$ of the same buffer solution was placed around the bag in a plastic beaker. To this, external solution quinidine and other substances were added as desired. The beaker was then covered tightly with Saran wrap and shaken mechanically at $25^{\circ} \mathrm{C}$ until equilibrium had been achieved. In practice this was found to occur within 18 hours.

Quinidine may be considered structurally as 4-hydroxymethyl-6-methoxy-quinoline connected by the secondary alcohol bridge to a quinuclidine ring. Therefore, not only was the effect of quinidine binding to albumin studied but also the binding to albumin of 4-hydroxymethyl6-methoxy-quinoline, ${ }^{4}$ 6-methoxy-quinoline, 4-hydroxymethyl quinoline, 5 quinoline, and naphthalene. The hydrochloride salt was used in all cases except that of naphthalene. These compounds were used as a means of evaluating the contribution of binding of the various parts of the quinoline molecule. In addition, the competitive or inhibitory effects of all of the above substances, except naphthalene, on quinidine-albumin binding were studied during the presence both of quinidine and of one of the other compounds in the system. The part played in the binding reaction by the quinuclidine

${ }^{1}$ Obtained from the Red Cross in solution form and from Pentex Co., Kankakee, Ill., in crystalline form. On paper electrophoresis both migrated as homogeneous peaks corresponding to albumin when barbital buffer $(\mathrm{pH} 8.6)$ and an ionic strength of 0.075 were used.

2 Obtained from Sharp and Dohme, division of Merck, Inc., Philadelphia, $\mathrm{Pa}$.

${ }^{3}$ A. H. Thomas Company, Philadelphia, $\mathrm{Pa}$.

4, 5 Synthesized with the generous aid of R. W. Greeff and Co., Nederlandsche Kininefabriek, Maarsen, Holland. 
TABLE I

Binding of quinidine and related compounds to albumin

\begin{tabular}{|c|c|c|c|c|c|c|c|}
\hline $\mathrm{B}^{*}$ & $\mathrm{C}_{q} \dagger$ & $\mathrm{C}_{\mathrm{B}} \mathrm{f}$ & & B & $\mathrm{C}_{\mathrm{q}}$ & $\mathrm{C}_{\mathrm{a}}$ & \\
\hline \multicolumn{4}{|c|}{ Quinidine } & \multicolumn{4}{|c|}{ 6-Methoxy-quinoline } \\
\hline $\begin{array}{r}62 \pm 3 \\
60 \pm 3 \\
55 \pm 2 \\
34 \pm 4 \\
28 \pm 1 \\
19 \pm 3 \\
9 \pm 2\end{array}$ & $\begin{array}{l}3.0 \times 10^{-6} \\
6.0 \times 10^{-6} \\
3.2 \times 10^{-5} \\
2.0 \times 10^{-4} \\
4.0 \times 10^{-4} \\
8.1 \times 10^{-4} \\
2.2 \times 10^{-3}\end{array}$ & $\begin{array}{l}2 \times 10^{-4} \\
2 \times 10^{-4} \\
2 \times 10^{-4} \\
2 \times 10^{-4} \\
2 \times 10^{-4} \\
2 \times 10^{-4} \\
2 \times 10^{-4}\end{array}$ & $\begin{array}{l}n \S=1.0 \\
K_{d} \|=1.3 \times 10^{-4}\end{array}$ & $\begin{array}{l}67 \pm 5 \\
66 \pm 2 \\
46 \pm 2 \\
36 \pm 2\end{array}$ & $\begin{array}{r}6.0 \times 10^{-6} \\
1.2 \times 10^{-5} \\
8.0 \times 10^{-4} \\
1.6 \times 10^{-3} \\
4 \text {-Hydro }\end{array}$ & $\begin{array}{l}2 \times 10^{-4} \\
2 \times 10^{-4} \\
2 \times 10^{-4} \\
2 \times 10^{-4} \\
\text { methyl-quin }\end{array}$ & $\begin{array}{l}n=6.1 \\
K_{d}=6.0 \times 10^{-4} \\
K_{a}=1.7 \times 10^{3}\end{array}$ \\
\hline \multicolumn{4}{|c|}{ 4-Hydroxymethyl-6-methoxy-quinoline } & $\begin{array}{l}42 \pm 4 \\
28 \pm 2 \\
18 \pm 2\end{array}$ & $\begin{array}{l}8.0 \times 10^{-6} \\
9.0 \times 10^{-4} \\
2.4 \times 10^{-3}\end{array}$ & $\begin{array}{l}2 \times 10^{-4} \\
2 \times 10^{-4} \\
2 \times 10^{-4}\end{array}$ & $\begin{array}{l}n=3.9 \\
K_{d}=9.0 \times 10^{-4}\end{array}$ \\
\hline $\begin{array}{l}73 \pm 4 \\
72 \pm 4 \\
50 \pm 2 \\
38 \pm 3\end{array}$ & $\begin{array}{l}5.5 \times 10^{-6} \\
1.0 \times 10^{-5} \\
3.6 \times 10^{-4} \\
7.0 \times 10^{-4}\end{array}$ & $\begin{array}{l}2 \times 10^{-4} \\
2 \times 10^{-4} \\
2 \times 10^{-4} \\
2 \times 10^{-4}\end{array}$ & $\begin{array}{l}n=2.9 \\
K_{d}=2.0 \times 10^{-4}\end{array}$ & \multicolumn{4}{|c|}{ Quinoline } \\
\hline \multicolumn{4}{|c|}{ Naphthalene } & $\begin{array}{l}50 \pm 2 \\
48 \pm 1 \\
40 \pm 3\end{array}$ & $\begin{array}{l}8.0 \times 10^{-6} \\
1.6 \pm 10^{-5} \\
80 \times 10^{-4}\end{array}$ & $\begin{array}{l}2 \times 10^{-4} \\
2 \times 10^{-4} \\
2 \times 10^{-4}\end{array}$ & $\begin{array}{l}n=8.1 \\
K_{d}=1.7 \times 10^{-3}\end{array}$ \\
\hline $\begin{array}{l}66 \pm 2 \\
65 \pm 2\end{array}$ & $\begin{array}{l}1.3 \times 10^{-4} \\
2.5 \times 10^{-4}\end{array}$ & $\begin{array}{l}2 \times 10^{-4} \\
2 \times 10^{-4}\end{array}$ & $\begin{array}{l}n=67.0 \\
K_{d}=6.6 \times 10^{-3} \\
K_{a}=1.5 \times 10^{2}\end{array}$ & $\begin{array}{l}34 \pm 3 \\
19 \pm 2\end{array}$ & $\begin{array}{l}1.3 \times 10^{-3} \\
5.4 \times 10^{-3}\end{array}$ & $2 \times 10^{-4}$ & $K_{a}=6.0 \times 10^{2}$ \\
\hline
\end{tabular}

* Per cent bound, with standard deviation.

$\dagger$ Free molar concentration of drug.

\$ Molar concentration of albumin.

$\$$ Number of areas available for drug binding per mole of albumin.

Dissociation constant of drug-albumin complex.

Tी Association constant of drug-albumin complex.

ring was evaluated in part through a study of the inhibitory effects of triethylene diamine (TED) and of benzyl alcohol on the quinidine-albumin interaction.

Quinidine concentration in protein and nonprotein solutions was determined in the Beckman DU spectrophotometer by the method of Josephson, Udenfriend and Brodie with ethylene dichloride as the extracting agent (6). The concentrations of 4-hydroxymethyl-6-methoxyquinoline, 6-methoxy-quinoline, 4-hydroxymethyl-quinoline, quinoline, and naphthalene were determined in a similar fashion. However, the concentration of the first two compounds, as well as that of quinidine, was determined at $250 \mathrm{~m} \mu$, while that of the 4-hydroxymethylquinoline and quinoline was determined at $235 \mathrm{~m} \mu$ and that of naphthalene at $220 \mathrm{~m} \mu$. In those experiments in which use was made of two compounds, both having an appreciable reading at the same wave length, the compounds were first separated on an acid alumina (Woelm) column with a solvent phase consisting of increasing ( 0.5 and 4.0 per cent) concentrations of ethanol in ethylene dichloride. Simultaneous recovery experiments were carried out following the addition of measured amounts of these various compounds both to the albu$\min$ and to the buffer solution of the dialysis system. If the recovery was not $100 \pm 10$ per cent, the experimental data were discarded. Recoveries from the column were $85 \pm 5$ per cent. From the concentrations of quinidine (or other compounds) measured on the two sides of the dialysis membrane, the free and bound fractions were calculated after a minor correction for the Donnan effect of protein.

The stoichiometric relationships and the dissociation constants of the drug-albumin complexes were evaluated as follows. The determinations of the bound fraction of the drug were made after the addition to the system of amounts of quinidine and/or quinoline compound varying from 100 to $50,000 \mu \mathrm{g}$. The formula reproduced by Goldstein (7) was solved to obtain values for the unknowns, $K$ and $n$, which represent the dissociation constant and the stoichiometric relationships, respectively.

The formula is $\beta=\frac{1}{1+(K / n P)+(x / n P)}$ where $\beta$ equals the fraction of bound to total drug, $K$ equals the dissociation constant of the drug-protein complex, $x$ equals the molar concentration of free drug, $n$ equals the number of receptor locations in the albumin molecule for each drug molecule, and $P$ is the molar concentration of the protein, here albumin. From the experimental data, solutions for the two unknowns were achieved either through the use of simultaneous equations or through the graphic plotting described by Goldstein (7). The association constant was derived as the reciprocal of the dissociation constant. The standard free energy of binding in kilogram-calories was calculated in the usual way by the product of the $\log$ of the association constant and 1.36. Each mean value noted in the results represents the average of at least 6 individual determinations, usually 10 to 12 , and in some cases up to 30 . 
RESULTS

Stoichiometry and the dissociation constants of the quinidine-albumin complex. The stoichiometric relationship and the dissociation constants were obtained at $\mathrm{pH}$ values of 7.4 , although they have been obtained at other $\mathrm{pH}$ values in experiments reported in another communication (8). The mean value for amount of drug bound at various free concentrations, the actual dissociation constants and the stoichiometric value are shown in Table I. The calculated results indicate that there is one receptor area for quinidine in each mole of albumin $(n=1.04)$. The dissociation constant is $1.3 \times 10^{-4}$ at $\mathrm{pH} 7.4$, although from other studies it is known to vary inversely with $\mathrm{pH}$ (8). Expressed as an association constant, this value becomes $7.7 \times 10^{3}$ at $\mathrm{pH}$ 7.4. Thermodynamically, this gives a standard free energy of binding of about $5.3 \mathrm{~kg}$-cal per mole of quinidine.

The binding of quinidine to $\gamma$-globulin. To the extent that these solutions resemble plasma, the $K$ and $n$ values obtained at $\mathrm{pH} 7.4$ may be used to predict the protein-bound fraction of quinidine in the serum of patients given commonly employed doses of quinidine. With an average serum albumin concentration of $4 \mathrm{~g}$ per cent and a therapeutic serum-quinidine concentration of $5 \mathrm{mg}$ per L (total concentration) the predicted value for the bound fraction would be about 80 per cent. A nearly identical value $(0.78)$ has been reported to be the fraction of quinidine and also quinine normally bound in human serum $(9,10)$. The similarity of these figures suggested that quinidine is not bound to any significant extent by serum globulin and led us to test this hypothesis experimentally. As the data from the albumin experiments would suggest, the amount of quinidine bound to $\gamma$-globulin in a dialysis equilibrium system was not significantly different from zero.

The binding of 4-hydroxymethyl-6-methoxyquinoline to albumin. The results of the binding studies carried out with this drug are shown in Table I. The calculated $n$ indicates that there are three receptor locations for 4-hydroxymethyl6-methoxy-quinoline in each mole of albumin. The dissociation constant is $2 \times 10^{-4}$ and the association constant is $5 \times 10^{3}$. The calculated free energy of binding is $5.0 \mathrm{~kg}$-cal, only slightly less than that calculated for quinidine-albumin bind- ing. Consequently, by inference the quinuclidine ring contributes only slightly to the binding.

The binding of 6-methoxy-quinoline to albumin. Results for the interaction of 6-methoxyquinoline with albumin are shown in Table $I$. The results indicate that 6 moles of this drug are bound by each interacting molecule of albumin. The dissociation constant is $6 \times 10^{-4}$. The association constant is therefore $1.7 \times 10^{3}$, and the free energy of binding $4.4 \mathrm{~kg}$-cal.

The binding of 4-hydroxymethyl-quinoline to albumin. The results for the binding of this drug to albumin are shown in Table I. Calculations indicate four receptor locations for this drug in each molecule of albumin. The dissociation constant is $9 \times 10^{-3}$. This is equivalent to an association constant of $1.1 \times 10^{3}$. The free energy of binding is calculated at $4.1 \mathrm{~kg}$-cal.

The binding of quinoline to albumin. The results for the binding of quinoline to albumin are shown in Table I. They indicate that there are eight receptor areas for quinoline in the albumin molecule. The dissociation constant is $1.7 \times 10^{-3}$, equivalent to an association constant of $6 \times 10^{2}$. The calculated standard free energy of binding is $3.5 \mathrm{~kg}$-cal.

The binding of naphthalene to albumin. Because of the extremely low solubility of naphthalene in aqueous solutions it was impossible to vary the free concentration so as to determine with adequate accuracy either the number of albumin receptor areas for naphthalene or the dissociation constant for the reaction. From the figures for per cent naphthalene bound to albumin obtained under these restricting conditions an $n$ of about 67 and a dissociation constant of about $6.6 \times 10^{-3}$ were calculated. This is equivalent to an association constant of $1.5 \times 10^{2}$, indicating an approximate free energy value of $2.9 \mathrm{~kg}$-cal. Despite the recognized inadequacy of the data and therefore the uncertainty of the extrapolations, they are presented as matters of interest in that 1) the calculated free energy value is similar to that found for the association of albumin with non-polar substituents (personal communication from Dr. Fred Karush) and 2) the free energy value is in general agreement with that predicted indirectly from our other experiments.

The effect of quinoline compounds in inhibiting quinidine-albumin binding. In order to show 
that the several related quinoline compounds were actually combining at the same receptor location on the albumin molecule as quinidine, some studies were carried out with both quinidine and one quinoline compound present in the dialysis system. As described under Methods these substances were subsequently separated by column chromatography, and each compound was read at its proper spectral peak. On the basis of the previously determined $K$ and $n$ values for the quinoline compounds, an amount of each (one per experiment) was added to the system that would be expected to reduce quinidine binding very significantly under the existing experimental conditions, provided the two drugs competed for the same reaction area in the albumin molecule. From the measured concentrations of unbound quinidine and quinoline drug and from other pertinent data, a predicted value for the bound fraction of quinidine was calculated. ${ }^{6}$ These values

TABLE II

Effect of quinoline compounds on quinidinealbumin binding

\begin{tabular}{|c|c|c|c|c|c|}
\hline $\mathrm{C}_{\mathrm{d}} *$ & $\mathrm{C}_{q} \dagger$ & $\mathrm{B}_{\mathrm{d}} \ddagger$ & $B_{\text {eq }} \S$ & $\mathbf{B}_{\mathbf{q}} \|$ & $B_{\text {eqa }} \| B_{\text {eq }} * *$ \\
\hline
\end{tabular}

4-Hydroxymethyl-6-methoxy-quinoline

$6.4 \times 10^{-4} \quad 4.4 \times 10^{-5} \quad 40 \quad 41 \quad 27 \quad 23 \quad 54$

6-Methoxy-quinoline

$\begin{array}{lllllll}2.1 \times 10^{-3} & 4.4 \times 10^{-5} & 25 & 31 & 25 & 32 & 54\end{array}$

4-Hydroxymethyl-quinoline

$\begin{array}{lllllll}4.4 \times 10^{-3} & 4.4 \times 10^{-5} & 10 & 13 & 22 & 30 & 54\end{array}$

Quinoline

$\begin{array}{lllllll}3.2 \times 10^{-3} & 4.4 \times 10^{-5} & 23 & 25 & 31 & 32 & 54\end{array}$

* Molar free concentration of quinoline compound found experimentally.

† Molar free concentration of quinidine found experimentally.

$\ddagger$ Per cent of quinoline compound experimentally bound.

$\S$ Per cent of quinoline compound expected bound on basis of $K$ and $n$ in Table $\mathrm{I}$.

II Per cent of quinidine experimentally bound in presence of quinoline drug and albumin concentration of $2 \times 10^{-4} \mathrm{M}$.

T Per cent of quinidine expected to be bound in presence of the amount of bound quinoline drug noted experimentally in column three (see footnote ${ }^{6}$ in text).

** Per cent of quinidine predicted as bound if the quinoline drugs did not compete for albumin receptor or were not present in the solutions.

${ }^{6}$ Preliminary assumptions were made that not only the quinoline compound and quinidine were competing simultaneously for one reaction area in albumin but also that the dissociation constants obtained experimentally for the quinoline compound-albumin interactions
TABLE III

Effect of benzyl alcohol and triethylene diamine on quinidinealbumin binding

\begin{tabular}{|c|c|c|c|c|}
\hline \multicolumn{5}{|c|}{ Benzyl alcohol } \\
\hline $\mathrm{C}_{\mathrm{ba}} *$ & $\mathrm{C}_{\mathrm{q}} \dagger$ & $\mathrm{B}_{\mathrm{a}} \downarrow$ & Beq $\S$ & $\mathrm{pH}$ \\
\hline $\begin{array}{l}2 \times 10^{-3} \\
7 \times 10^{-3} \\
2 \times 10^{-2}\end{array}$ & $\begin{array}{l}7.0 \times 10^{-6} \\
7.2 \times 10^{-6} \\
1.0 \times 10^{-5}\end{array}$ & $\begin{array}{l}65 \pm 3 \\
58 \pm 2 \\
23 \pm 9\end{array}$ & $\begin{array}{l}62 \\
62 \\
62\end{array}$ & $\begin{array}{l}7.4 \\
7.4 \\
7.4\end{array}$ \\
\hline \multicolumn{5}{|c|}{ Triethylene diamine } \\
\hline $\mathrm{C}_{\text {TED }}$ & $\mathrm{C}_{\mathrm{q}}$ & $\mathbf{B}_{\mathbf{q}}$ & $\mathrm{B}_{\text {eq }}$ & $\mathrm{pH}$ \\
\hline $\begin{array}{l}1 \\
1\end{array}$ & $\begin{array}{l}7.0 \times 10^{-6} \\
8.0 \times 10^{-6}\end{array}$ & $\begin{array}{l}64 \pm 2 \\
58 \pm 4\end{array}$ & $\begin{array}{l}90 \\
62\end{array}$ & $\begin{array}{r}10.0 \\
7.4\end{array}$ \\
\hline
\end{tabular}

* Molar concentration of benzyl alcohol.

† Molar free concentration of quinidine.

$\ddagger$ Per cent of quinidine bound, with standard deviation. $\S$ Per cent of expected bound quinidine based on values from Table I.

|| Molar concentration of triethylene diamine.

and the experimentally-obtained values were then compared with each other and with the predicted value for bound quinidine in the absence of a competing drug. The results are shown in Table II. The addition of sufficient 4-hydroxymethyl6-methoxy-quinoline to the system to achieve a $6.4 \times 10^{-4} \mathrm{M}$ concentration of free drug reduced

were applicable to interaction at this one location. The predicted value for the fraction of quinidine bound in the presence of other drugs was then obtained as follows. The fraction of quinoline (competing) drug found to be bound experimentally (column 3, Table II) gives an equation relating the concentration of bound drug to the total drug concentration in the albumin solution. This relationship was used with a second equation relating the measured concentration of unbound or free quinoline to the concentration of bound drug and to the total concentration. In the case of 4-hydroxymethyl-6-methoxyquinoline, 0.40 total equals bound, and total equals $6.4 \times$ $10^{-4}$ plus bound. Solution of the simultaneous equations gives a value for the concentration of drug bound to albumin. This concentration divided by the number of protein receptor areas for the drug gives the concentration of drug bound at the one location at which quinidine may attach or compete. This result then presumably gives the concentration of protein receptor areas covered by competing drug and not available for quinidine binding-4.27 $\times 10^{-4} / 2.9\left(\mathrm{~B}_{\mathrm{c}} / n\right)$ equals $1.47 \times 10^{-4}$. The difference between this value and the total available areas $\left(\mathrm{P}_{\mathrm{c}}\right.$ or $2 \times 10^{-4} \times n$ or 1$)$ gives the concentration of protein receptor locations still available for quinidine-albumin interaction, $\left(2 \times 10^{-4}-1.47 \times 10^{-4}\right.$ equals $\left.0.53 \times 10^{-4}\right)$. By substitution of this value, along with the appropriate $K, n$, and $\mathrm{F}_{\mathrm{c}}$ for quinidine, in the Goldstein equation a predicted value for the bound fraction of quinidine is obtained (column 6, Table II). 
the percentage of quinidine bound (unbound quinidine concentration of $4.4 \times 10^{-5} \mathrm{M}$ ) from 54 to 27 , close to the predicted value of 23 per cent. With a concentration of free 6-methoxyquinoline of $2.1 \times 10^{-3}$ nearly the same result was achieved, a reduction in the quinidine bound from 54 to 25 per cent. This may be compared with the predicted result of 32 per cent bound. A reduction of the bound fraction of quinidine from 54 to 22 per cent was achieved with a $4.4 \times 10^{-3}$ $M$ concentration of 4 hydroxymethyl quinoline. The 22 per cent figure is similar to the predicted value of 30 per cent. A free quinoline concentration of $3.2 \times 10^{-3} \mathrm{M}$ reduced the fraction of quinidine bound to albumin from 54 to 31 per cent, almost exactly as predicted. The data consequently provide strong evidence that there is competition between quinidine and the related compounds tested for one specific reactive area in albumin.

The effects of triethylene diamine and benzyl alcohol on quinidine binding with albumin. An attempt to learn something further about the importance of the quinuclidine ring and the alcoholic $\mathrm{OH}$ site to the binding reaction was carried out through the addition of varying amounts of TED or of benzyl alcohol along with quinidine to the dialysis system. Addition of benzyl alcohol, which presumably might compete for an albumin site with the combined quinuclidine ring and adjacent alcohol fragment of quinidine, led to the results indicated in Table III. In the presence of free quinidine, concentrations of slightly less than $1 \times 10^{-5} \mathrm{M}$ benzyl alcohol in $2 \times 10^{-3} \mathrm{M}$ concentrations had little effect on binding. In the presence of a $7 \times 10^{-3} \mathrm{M}$ alcohol concentration, bound quinidine was reduced slightly from the 62 per cent expected if no alcohol were present to 58 per cent. With concentrations of benzyl alcohol at $2 \times 10^{-2} \mathrm{M}$ the inhibition of quinidine-albumin binding was very marked. An average of only 23 per cent was calculated for the bound fraction as compared with an expected 62 per cent. This is compatible with a contribution by the quinuclidine ring and the secondary alcohol of something like $1.5 \mathrm{~kg}$-cal to the total energy of binding, a value close to that predicted from the previously noted experimental results. On the other hand, TED which presumably is similar to the basic quinuclidine structure, failed to reduce ap- preciably quinidine binding at $\mathrm{pH} 7.4$ even when present in concentrations as great as $1 \mathrm{M}$. Only a slight reduction from an expected value of 62 per cent to a calculated value of 58 per cent was found with $1 \mathrm{M}$ concentrations. Since the two pK's of triethylene diamine are 5 and 8.4 , at $\mathrm{pH}$ 7.4 one of the nitrogens is mainly positively charged, as is the nitrogen in the quinuclidine ring (pK, 8.6). Therefore, further evidence is adduced that the quinuclidine ring per se does not seem to play an important part in the binding of quinidine to albumin, at least when the nitrogen group is positively charged, but that the alcoholic bridge fragment contributes something like 1 $\mathrm{kg}$-cal to the reaction.

In view of these conclusions, we were somewhat surprised to find that in the presence of carbonate buffer at $\mathrm{pH} 10$, the same concentration of TED reduced the binding to 64 per cent from an expected 90 per cent. This significant reduction in the binding, however, occurs at a $\mathrm{pH}$ at which the two nitrogens in the triethylene diamine and the nitrogen of the quinuclidine ring of quinidine are essentially all noncharged. We have found that at this $\mathrm{pH}$ the increased binding, found normally, relates mainly to an increase in the number of quinidine receptor areas in albumin from 1 to 3 (8), possibly because of the loss of the polar effect of the positively charged nitrogen. This increased $n$ value (3.0) is the same as that calculated for 4-hydroxymethyl-6-methoxy-quinoline. It is suggested that neutral TED in $1 \mathrm{M}$ concentration may compete with neutral quinidine for these additional two albumin binding areas.

\section{DISCUSSION}

These data reveal a moderately large $\left(K_{a} 7.7 \times\right.$ $10^{3}$ ) association constant for the quinidine-albumin reaction, and an uncommon, one-for-one molar interaction-i.e., one receptor area for quinidine attachment in each albumin molecule. The basic quinoline ring structure apparently contributes a major portion of the binding energy, about 2.9 out of $5.3 \mathrm{~kg}$-cal. The neutral nitrogen of the quinoline ring $(\mathrm{pK}, 4.0)$, the methoxy group, and the secondary bridging alcohol group also contribute to the energy of binding and therefore presume to be sites of interaction with 
the albumin molecule. ${ }^{7}$ The specificity of the quinidine-albumin reaction is contributed to by all the three reactive groups as is evident by the increasing number of binding areas in albumin involved and the decreasing association constant values resulting as these groups are deleted from the molecule. Either hydrogen bonding or Van der Waals forces could account for these interactions. Formation of hydrogen bonds seems more probable in view of the virtually identical binding energies ( 0.5 to $1.0 \mathrm{~kg}$-cal) concerned with each of the three reactive sites, and particularly because the experimental data appear to show that the energy of binding is the same for all albumin locations involved in binding both quinidine and related quinoline compounds. Equal Van der Waals interactions in all cases would imply nearly identical spatial complementarity of albumin and drug at the critical areas. It is improbable that the eight areas concerned in albumin would have the required structural identity. On the other hand, albumin sites at which hydrogen bond linkage to the quinoline nitrogen, the methoxy, and the hydroxymethyl groups of quinidine might occur have not been identified. Our recent unreported studies are compatible with the concept that the quinuclidine nitrogen forms a hydrogen bond with an imidazole nitrogen in a histidine residue of albumin. The methoxy group may form a hydrogen bond with a hydroxyl group in serine, and

T The term site as commonly used in characterizing drug-protein interaction has at least two connotations and needs to be defined for purposes of the present discussion. It has been used to refer to the point of molecular interaction between a reactive group or atom in the drug and a corresponding group in an amino acid of protein. Electrostatic interaction between a positively charged nitrogen atom in a drug and a negatively charged hydroxyl group in protein is one such example. We have reserved site for use in describing this form of interaction. Site or receptor site has also been commonly used to refer to the general area at which a drug is attached to a protein, irrespective of whether one or many drug-protein reactive group interactions take place, or whether, in our terminology, one or many sites of interaction are involved. Thus, in common parlance $n$ is defined as the number of drug receptor sites per molecule of protein. In order to avoid needless confusion between the former (single) and the latter (in this case, multiple interaction), we have arbitrarily substituted the terms area or location for use in the second connotation. We have defined $n$ as the number of receptor locations (or areas) for drug attachment to the albumin molecule. the suggestion has been made that a similar reaction may link the secondary alcohol to a hydroxyl group in tyrosine (personal communication from Dr. I. M. Klotz).

At $\mathrm{pH} 7.4$, the quinuclidine ring does not seem to play a very great part in the binding of quinidine to albumin. Its most important role may relate to the action of the charged nitrogen in preventing binding at more than one reactive area in albumin. This role is suggested 1) by the fact that the simpler compound without the quinuclidine ring - 4-hydroxymethyl-6-methoxy-quinoline-appears to react at three binding areas in albumin and 2) by the fact that when the positive nitrogen in the quinuclidine ring becomes neutral with increasing $\mathrm{pH}$, the number of receptor areas for quinidine in albumin is also three. In brief, the uncharged form of quinidine may be able to attach itself at two additional areas in albumin where the charged form is unable to penetrate or attach. While this hypothesis may appear more attractive, an additional consideration is deserving of mention-competition for quinidine between the water and protein phases. Water solubility of quinidine varies markedly with $\mathrm{pH}$ in an inverse fashion. This relationship might be anticipated if the charged nitrogen groups (pK's of 4.0 and 8.6) were undergoing polar interactions with water. At a $\mathrm{pH}$ of 10 , quinidine is nearly all in the uncharged form, quinidine solubility is slight, and with increasing $\mathrm{pH}$, it rapidly becomes negligible. With decreasing $\mathrm{pH}$ below 7 , quinidine solubility increases markedly, the acidic salts-quinidine hydrochloride and quinidine sulfate-being the compounds commonly used to prepare concentrated aqueous solutions of quinidine. Quinidine's high aqueous solubility at low $\mathrm{pH}$, reflecting predominance of the most polar forms of the molecule, might be partially responsible for the reciprocally low degree of attachment to protein we have observed in unreported studies. In contrast, low aqueous solubility of the neutral form of the drug at high $\mathrm{pH}$ may account partially for the marked drug affinity for albumin noted under these conditions. To the extent that this process is operative, a plot of quinidine binding by albumin against $\mathrm{pH}$ should resemble a quinidine acid titration curve. Unfortunately, the former plot as obtained experimentally is more complex than the 
latter. Interpretation is not so simple as proposed and the issue remains unresolved.

The similarity of association constants for the quinidine-albumin and the (4-hydroxymethyl-6methoxy-quinoline)-albumin reactions, and the finding of a receptor area in albumin available to both drugs led us to test whether these drugs have any common cardiovascular effects (11). The substituted quinoline does have a peripheral vasodilation action like that of quinidine. The hypotension produced by both drugs is similarily reversed by sympathomimetic amines. Finally the effective "hypotensive" dose of 4-hydroxymethyl-6-methoxy-quinoline is three or four times that of quinidine, a relationship predictable by translation of the relative quantitative aspects of the drugs binding with albumin to their binding via a similar hypothetical receptor in arteriolar protein. A comparison of the action of the two drugs on the heart gives a different picture. The substituted quinoline compound possesses minimal antiarrythmic and apparently none of the usual myocardial depressant properties of quinidine. The indirect implication is that the pharmacologic effects of quinidine on the heart are dependent on the presence of the quinuclidine ring. From these considerations two questions are posed, neither of which is currently answerable except in an indirect and speculative fashion.

The first question is concerned with the extent to which the characteristics of quinidine-albumin interaction can be considered to reflect the nature of quinidine binding by cell protein. Studies such as those of Wegria and Boyle (12) tell us simply that there must be a considerable amount of cellular quinidine binding to protein. We are aware of only one quantitative study concerning quinidine binding to isolated purified cell protein. This is one of our unreported studies involving the use of Escherichia coli endotoxin, a material thought to be membrane lipoprotein although perhaps modified in preparation. From the results of that study we calculated that the number of receptor areas for quinidine per molecule of endotoxin (based on an estimated $10^{6}$ molecular weight of endotoxin) is about 1.5. While this $n$ value is similar to that for the reaction between quinidine and albumin, the association constant at $\mathrm{pH} 7.4$ is approximately 100 -fold greater, being $8.0 \times 10^{5}$. It may well turn out that quinidine is highly associated with certain fractions of cell protein and negligibly associated with others. Our findings of an apparently rather specific interaction between quinidine and albumin and a negligible or absent one between quinidine and $\gamma$-globin makes this speculation plausible.

The second question is: Why should the presence of the quinuclidine ring be critical to the cardiac but not to the peripheral vascular action of quinidine? The cinchona alkaloids have been known for many years to have the properties of a surface film agent. Accordingly, the cardiac cell surface has been proposed as a critical site with respect to quinidine action. The results indicating a quinidine-induced alteration in ionic transfer rates and ion distribution are compatible with this concept (1-4). Other surface active agents derive their activity from the hydrophilic nature of one portion of the molecule and a nonaqueous phase affinity of another. The substance is thus molecularly oriented so as to link aqueous and nonaqueous phases. From the observed characteristics of quinidine-albumin binding and of quinidine solubility in water, it appears that under appropriate circumstances quinidine can play this role. The quinoline portion of the molecule is albuminophilic and binds to protein, while the charged quinuclidine ring is hydrophilic and interacts with water. If this kind of reaction were required at the cell membrane interfaces in order to bring about the myocardial effects of quinidine, the necessary presence of the charged quinuclidine ring would be explained. We should therefore like to suggest the possibility that the cardiac effects of quinidine can be at least in part explained by its molecular orientation at cell interfaces.

\section{SUMMARY}

The quantitative aspects of quinidine binding to albumin were investigated in an in vitro dialysis equilibrium system. Similar investigations were made using related compounds-4-hydroxymethyl-6-methoxy-quinoline, 6-methoxy-quinoline, 4-hydroxymethyl-quinoline, quinoline, and naphthalene. The inhibitory effects on quinidine-albumin binding of these latter drugs, save naphthalene, 
and the effects of benzyl alcohol and triethylene diamine were evaluated. Quinidine- $\gamma$-globulin binding was also evaluated and found to be negligible.

The quinidine-albumin reaction is characterized by the presence of one receptor area for quinidine per protein molecule and a moderately large association constant $\left(7.7 \times 10^{3}\right)$. The interaction of albumin and the quinoline compounds related to quinidine is characterized by an increasing number of available binding sites in albumin and decreasing association constants as the chemically active sites in the quinoline molecule are eliminated. All the quinoline drugs tested appear to compete with quinidine for a presumed common receptor area in albumin. This competition occurs in a manner which is at least semiquantitatively predictable from quinoline relationships with albumin, relative to those of quinidine with albumin.

Quinidine binding by albumin seems to depend upon protein interaction with the basic quinoline ring and a more or less equal interaction with the quinoline nitrogen, the methoxy, and the secondary alcohol groups. The last three groups probably interact with protein sites as a result of hydrogen bond formation. Minor interaction of the quinuclidine ring structure and albumin could not be excluded.

Observations on the similarities of 4-hydroxymethyl-6-methoxy-quinoline and of quinidine binding to albumin, on their similarities of structure, and on their comparative effects on the cardiovascular system may be considered in agreement with two hypotheses. Quinidine-albumin binding reflects the nature of the combination of quinidine with a "critical" receptor in the protein of arteriolar smooth muscle. Cardiac action of quinidine may result from a special orientation of the molecule at interfaces, with binding of the quinoline ring to interface lipoprotein and of the charged quinuclidine ring to water.

\section{ACKNOWLEDGMENT}

The authors wish to express their thanks to Dr. Fred Karush and Dr. I. M. Klotz for advice during the course of these studies, and to Dr. Karush for criticisms of the manuscript. The authors also wish to acknowledge with thanks the technical assistance of Miss Eve Kritcher and Miss Jean Cannon.

\section{REFERENCES}

1. Holland, W. C. A possible mechanism of action of quinidine. Amer. J. Physiol. 1957, 190, 492.

2. Holland, W. C., and Klein, R. L. Effects of temperature, $\mathrm{Na}$ and $\mathrm{K}$ concentration and quinidine on transmembrane flux of $\mathrm{K}^{42}$ and incidence of atrial fibrillation. Circulat. Res. 1958, 6, 516.

3. Conn, H. L., Jr., and Wood, J. C. Quinidine and digitalis effects on cation kinetics and circulation of the isolated heart. Fed. Proc. 1958, 17, 28.

4. Conn, H. L., Jr., and Wood, J. C. Acute effects of quinidine on $\mathrm{K}$ exchange and distribution in the dog ventricle. Amer. J. Physiol. 1960, 199, 151.

5. Uyeki, E. M., Geiling, E. M. K., and Dubois, K. P. Studies on the effects of quinidine on intermediary carbohydrate metabolism. Arch. int. Pharmacodyn. 1954, 97, 191.

6. Josephson, E. S., Udenfriend, S., and Brodie, B. B. Estimation of basic organic compounds in biological material. VI. Estimation by ultraviolet spectrophotometry. J. biol. Chem. 1947, 168, 341.

7. Goldstein, A. The interactions of drugs and plasma proteins. Pharmacol. Rev. 1949, 1, 102.

8. Conn, H. L., Jr., and Luchi, R. J. Some studies on the nature of quinidine-albumin interaction ( $a b-$ stract). J. clin. Invest. 1960, 39, 978.

9. Davis, B. D. The binding of chemotherapeutic agents to proteins and its effect on their distribution and activity in Symposium of Section on Microbiology, N. Y. Acad. Med., no. 2. New York, Columbia Univ. Press, 1949, p. 44.

10. Hiatt, E. P., and Suhrie, V. Renal excretion of cinchona alkaloids and some quaternary base derivatives and their effect on renal hemodynamics. Fed. Proc. 1946, 5, 46.

11. Conn, H. L., Jr., Helwig, J. H., and Luchi, R. J. The circulatory effects of a quinidine homolog, 4 hydroxymethyl 6 methoxy quinoline. Physiologist $1960,3,39$.

12. Wegria, R., and Boyle, M. N. Correlation between the effect of quinidine sulfate on the heart and its concentration in the blood plasma. Amer. J. Med. 1948, 4, 373. 